\title{
Vagueness, Ambiguity, and Underspecification
}

\author{
Manf red Pinkal \\ University of the Saarland, Germany
}

\section{Introduction}

At the beginning of natural-language semantics as a formal discipline, there was the insight that the notion of meaning and the notion of truth are narrowly related. If we know the meaning of a sentence, we know whether it is a truthful or false description of a given state of the world. Meaning information contained in a sentence can therefore be modelled in terms of truth conditions: functions from possible states of the world to the truth values $\mathrm{T}$ and $\mathrm{F}$. The meaning of non-sentential expressions is indirectly, but unequivocally related to the sentence meaning by virtue of the principle of compositionality. The picture is an idealization, of course: Compositionality is not an empirical fact, but a methodological assumption. Its appropriateness and limitations have been intensively disputed. Also, identification of meaning with truth conditions abstracts away from important facets of linguistic meaning. The anaphoric potential, as a special case of the context-changing force of natural-language expressions, is the best-investigated and most intensively discussed non-truth-conditional aspect of meaning.

Another problem arises from the requirement that either truth or falseness must be assigned to all sentences with respect to every state of the world. Word meaning often is so imprecise or underspecified that there is no clear-cut boundary between true and false. The vagueness phenomenon is pervasive in natural languages: Almost every natural-language concept has some margin of imprecision in at least some cases of application. Prototypical vague expressions - colour terms like red and blue, scalar adjectives like tall and clever, spatial adverbs and prepositions - come with a substantial borderline-area of semantic indefiniteness in any natural context (although the size and location of these areas may vary). The vagueness phenomenon has never gained greater interest in linguistics, however. It seems to be more of a philosophical matter: A very basic and important problem, which needs to be carefully thought about, but which does not interact a lot with the rest of natural-language meaning structure. In fact, most of the research on vagueness has been done in the borderline area of linguistics and philosophy.

During the last years, however, interest has been growing in matters which are narrowly related to the semantics of vague expressions. This interest arose neither in theoretical linguistics nor in philosophy of language, but in the application oriented field of computational semantics. It emerged from the problem of a proper treatment of ambiguous expressions.

Natural language expressions tend to be multiply ambiguous. Sentences do not have truth conditions per se, but only under a certain reading. In the field of natural-language understanding, the task is to determine what a sentence does mean in a given context of utterance rather than just what it could mean in general. Among theoretical linguists, a simple process model of utterance understanding is popular: the linguistic component generates the set of all possible readings, and then a non-linguistic resolution procedure filters out all but the one reading intended by the speaker of the utterance. This model is neither cognitively plausible nor computationally feasible. There is a need for representation formalisms which allows to avoid the generate-and-test solution, i.e., formalisms which represent the range of possible readings in a more compact way than by enumeration of alternative interpretations. Several such formalisms for underspecified semantic 
information ("underspecification formalisms", in short) have been proposed during the last years. They are similar in their basic approaches, describing the range of possible readings in terms of a constraint set, and modelling the resolution process as a stepwise, monotonic extension of this constraint set, which corresponds to the subsequent reduction of the range of possible readings. Also, these approaches cover comparable ranges of phenomena: local cases of underspecification (arising, e.g., from lexical ambiguities) as well as global, structural cases like scope underspecification.

It is an interesting question what the underspecified representations really mean, beyond the fact that they stand for sets of possible readings. Can they be assigned denotations directly, and in which way are these related to the denotations of the members in the corresponding disambiguation set? The question is interesting in itself, for one would like to have a precise notion of what meaning representations mean. However, there is a more substantial motivation to provide underspecified representations with a direct semantics. The final result of the utterance understanding process in a discourse is quite often not a fully disambiguated reading, and in many cases this is not perceived as a flaw, nor does it render the exploitation of the utterance information impossible. Full disambiguation may not even substantially improve the usefulness of the utterance information, in certain cases.

\section{Most researchers in the Saarbrücken computational linguistics group work on some underspecification problem}

If you are interested in underspecification, and are told sentence (1), you may conclude that it is a good idea to contact the Saarbrücken group, irrespective of whether it is the same or different problems the group members work on, and whether they do their work collectively or independently. Drawing this conclusion involves reasoning on the basis of underspecified meaning representations. Of course, one could do the reasoning on underspecified representations in terms of applying standard deduction techniques to each of the readings it covers, and compare the results. However, to use underspecified representations in the process of semantic analysis, and to generate all readings out of them afterwards in order to apply standard deduction in parallel is unnatural and inefficient again. There is a need for deduction techniques that directly work on underspecified representations. Accordingly, there is a need for an entailment concept and a direct denotational semantics for underspecified representations, in order to guide the development of deduction techniques and to control their performance.

The basic problem for underspecified ambiguous expressions is similar to the vagueness problem mentioned above: There are cases where it is impossible to definitely assign truth or falseness to a sentence, because of underspecified meaning information. Of course, the vagueness of red has a different status from, say, the indefiniteness caused by the ambiguity of bank, or by the failure of assigning a pronoun a unique reference. However, vagueness and ambiguity-related underspecification share the above-mentioned basic property: the impossibility of definite truth-value assignment. Since the treatment of vague expressions in a truthconditional framework has been thoroughly investigated through the last decades, it may be worth having a look into the arguments and solutions proposed in that field. The author of this paper tried.to provide a general account of all kinds of vagueness and ambiguity phenomena, including a formalism which allows to describe contextual refinement or "precisification", in his former work on vagueness ([Pinkal 1985] and [Pinkal 1995])!.

I will give a short report of U. Reyle's Underspecified Discourse Representation Theory (UDRT) in the next section, as a representative of the family 
of currently available underspecification theories. In Section 3, the relevant features of a denotational interpretation for indefinite expressions are sketched, with semantic covariation as the crucial concept. In Section 4, I will try to point out the relation between the two approaches, outline an appropriate concept of partial interpretation for underspecified representations, and indicate a solution to the problem of structural covariation between underspecified representations. In the final section, I will address the question how the difference between vagueness and ambiguity is related to the level-distinction between representational underspecification and denotational indefiniteness.

\section{Underspecification formalisms}

During the last years, several formalisms for the representation of underspecified semantic information have been proposed. ${ }^{2}$ These formalisms are rather similar in their descriptive force and in the kind of the formal devices they use. A common feature is the use of meta-variables which range over expressions of the semantic representation language. A trivial example is given below, where (3) is an underspecified representation for the lexically ambiguous sentence (2), specifying the two specific readings in (4).

Bill owns a pen

$$
\begin{aligned}
& \left\{\exists x(X(x) \& \text { own }(b, x)), X \in\left\{\operatorname{pen}_{1}, \operatorname{pen}_{2}\right\}\right\} \\
& \left\{\exists x\left(\operatorname{pen}_{1}(x) \& \text { own }(b, x)\right), \exists x\left(\operatorname{pen}_{2}(x) \& \text { own }(b, x)\right)\right\}
\end{aligned}
$$

The representation task is simple here, as it is in relational ambiguities (like John's book) or simple cases of referential ambiguities, since the source of underspecification is a certain local position in the otherwise unambiguously given semantic structure. The position is marked by a meta-variable, and the specific readings are obtained by substituting the meta-variable with its possible instantiations. This technique is all but new, of course: e.g., unbound variables have always taken to be a natural device to represent referential underspecification. Structural underspecification, as it is induced by scope ambiguities, is more difficult to handle because it concerns global differences in semantic structures. Therefore underspecification cannot be restricted to some local part of the representation.

All of the currently available underspecification formalisms offer a treatment of this problem. The solutions look different, but are essentially similar. As an illustrating example, I give an analysis of a scope-underspecification example in the style of Reyle's UDRT. I use standard predicate-logic rather than DRT for the representation of object-level semantic information, for the sake of readability. The design of the description language formalism and the choice of an object-level semantics are basically independent of each other.

Every student didn't pay' attention

$$
\begin{aligned}
& \{\forall \mathrm{x}(\operatorname{student}(\mathrm{x}) \rightarrow \neg \text { pay_attention }(\mathrm{x})), \\
& \neg \forall \mathrm{x}(\operatorname{student}(\mathrm{x}) \rightarrow \text { pay_attention }(\mathrm{x}))\}
\end{aligned}
$$




$$
\left\{\begin{array}{r}
\mathrm{l}_{1}: \forall \mathrm{x}\left(\operatorname{student}(\mathrm{x}) \rightarrow \mathrm{l}_{2}\right), \mathrm{l}_{3}: \neg \mathrm{l}_{4}, \mathrm{l}_{0}: \text { pay_attention }(\mathrm{x}), \\
\left.\mathrm{l}_{1} \leq \mathrm{l}_{\mathrm{T}}, \mathrm{l}_{3} \leq \mathrm{l}_{\mathrm{T}}, \mathrm{l}_{0} \leq \mathrm{l}_{2}, \mathrm{l}_{0} \leq \mathrm{l}_{4}\right\}
\end{array}\right.
$$

Sentence (5) is ambiguous between the readings in (6), (7) is the corresponding underspecified representation. The representation uses "labels" $l_{i}$ as meta-variables. They go into partially specified semantic structures like $\forall x$ (student $(x) \rightarrow l_{2}$, as in the simple case (3). But they are also used to name complex structures and express relational constraints between different sub-structures. " $\leq$ " is a partial ordering relation standing for "subordination"; $l_{i} \leq l_{j}$ says that the structure labelled with $l_{i}$ is subordinate or simply a (proper or improper) part of the structure labelled with $\mathrm{l}_{j}$. The labelling operator ":" can be read as equation between a label and a partial or full structural description. The designated label $\mathrm{l}_{\mathrm{T}}$ stands for the representation of the whole structure. The readings described by a constraint set are its minimal solutions, i.e., the smallest object-language representations which simultaneously satisfy all of the constraints. In the case of (7), the solutions correspond to the possible linear orderings of scope-bearing expressions. There are two of them, each of which corresponds to one of the formulae in (6).

Constraint systems like UDRT allow to model the process of disambiguation by monotonic extension of the description. E.g., disambiguation of (5) to one of the readings in (6) is expressed by alternatively adding $l_{3} \leq l_{2}$ or $l_{1} \leq$ $\mathrm{l}_{4}$ to the constraint set. Thus there is no need to generate completely specified readings as input to the resolution process. In our specific example, there is just one step of disambiguation possible. However, it is not in general required that disambiguation is done at once and completely. Stepwise addition of constraints may lead to a sequence of representations which express subsequent stages of partial disambiguation. Moreover, because of the declarativity of the formalism, there is no need for a strict sequential ordering between linguistic analysis and resolution. The formalism is basically compatible with interleaved or incremental architectures.

As mentioned in Section 1, underspecification formalisms are not only supposed to act as notational devices for a natural and efficient process model of semantic analysis, but they should also support direct reasoning with underspecified information. This, in turn, makes a denotation-based entailment concept highly desirable. I will finish this section by giving a short account of some "simple" solutions for the interpretation of underspecified structures which are based on the treatment of meaning alternatives as disjunctions.

If Sentence (3) is uttered in a context where both readings of pen are possible interpretation alternatives, the hearer will take the sentence to be true, if only one of the readings corresponds with the facts, which fits well to the interpretation of ambiguity as disjunction.

\section{Some student takes every course in linguistics}

Similarly, if (8) is uttered in a situation where all students take some course, but not all take the same one, the hearer is likely to accept the sentence as true on the basis of its wide (narrow-scope existential) reading, and to insist in the fact that there is an alternative narrow reading which would render it true. This also seems to support the disjunction interpretation, since the wide reading is equivalent to the disjunction of the alternatives. Consider, however, Sentences (9) and (10).

John doesn't own a pen.

\section{It is not the case that some student takes every course in linguistics}


(9) becomes already false according to de Morgan's Law, if one of the readings does not apply, when analyzed as negation of the disjunction of its readings. (10) correspondingly turns out to be equivalent with the negation of its wide reading, i.e., its narrower interpretation alternative, which is a definitely counterintuitive result. The disjunctive interpretation goes nicely hand in hand with the pragmatic principle of charity, in plain affirmative cases like (3) and (8): If the hearer has the choice between different options to interpret an utterance, he will make his decisions in a way that the utterance expresses a true proposition. In the scope of a negation, however, the disjunction analysis leads to results which are contrary to the hearer's behaviour. They always correspond to the maximally incooperative choice of a reading.

There is an alternative way of a disjunction analysis, however. So far, I have assumed that the disjunction is locally introduced by the underspecified part of the meaning representation, and may be embedded in the scope of other operators. Alternatively, one can take the semantic value of an underspecified expression to be equivalent to the global disjunction of its readings. This solves the problem with (9) and (10): $\neg \mathrm{A}$ will be interpreted as $\neg \mathrm{A}_{1} \vee \neg \mathrm{A}_{2}$, which again nicely conforms to the cooperative hearer's behaviour. The wide-scope disjunction treatment is adopted by [Reyle 1993]. Reyle defines an entailment concept on this basis, and sets it to work in a deduction system for underspecified representations. But there are general adequacy problems also with the wide-scope disjunction analysis. First, look at cases of non-assertional utterances like (11).

\section{Does John own a pen?}

In a situation where (3) is true under one, but not under the other disambiguation, the disjunction analysis predicts an affirmative answer to (11), if the question operator takes scope over the disjunction. This is hardly adequate, given that the hearer does not know about the reading intended by the speaker. Concerning the wide-scope variant, it is completely unclear, however, how a disjunction between questions should be interpreted at all. Another problem is that the wide-scope disjunction treatment leads to a much too permissive notion of entailment. A set of premisses entails a conclusion, if every possible reading of the the premisses entails at least one possible reading of the conclusion. Now, take $A$ to be ambiguous between two mutually exclusive readings, as in the case of the homonymous predicate pen. As shown in (12), it holds that A entails $\neg \mathrm{A}$, because each reading of A excludes the other, which is doubtlessly not a desirable result.

$$
A \Leftrightarrow A_{1} \vee A_{2} \mid=\neg A_{1} \vee \neg A_{2} \Leftrightarrow \neg A
$$

Whichever special policy is taken, the interpretation of underspecified expressions in terms of classical disjunction is unsatisfactory. In the area of vagueness research, this has been a common-place truth for a long time already. As Max Black points out in his classical essay, "the finite area of the field of application of the word is a sign of its generality, while its vagueness is indicated by the finite area and lack of specification of its boundary" ([Black 1937]:432). In order to model the "lack of specification" in a denotational semantics, some extension of classical standard semantics is required. Various proposals for such an extension have been made in the field of vagueness research. In the following section, I will inspect and comment the major alternatives. 


\section{The interprētation of vague expressions}

Vagueness, as well as ambiguity, has the effect that in certain cases none of the truth values "true" and "false" can be definitely assigned. There are two basic alternatives for modelling this property in a truth-conditional framework. The range of possible truth-values (e.g., by I for "indefinite") can be extended, or truth functions denoted by natural-language sentences can be taken to be partial functions, only. The first strategy leads to many-valued logics (esp. three-valued ones), the second one gives rise to so-called super-valuation semantics.

The basic task for three-valued logics is to extend the interpretation of logical operators to the third truth value in an appropriate way. There are quite a lot proposals for three-valued predicate logics around, but the interesting ones coincide in their interpretations of conjunction and disjunction. ${ }^{3}$ The strategy followed is to assign a conjunction (disjunction) one of the definite values " $T$ " and " $F$ ", if that truth-value is uniquely determined by the definite values of the conjuncts (disjuncts); otherwise, it receives the indefinite value "I". Accordingly, A \& B is true if both of the conjuncts are true, false, if at least one of the conjuncts is false, and indefinite otherwise; the truth functions for conjunction, and the corresponding one for disjunction are shown in (13).

\begin{tabular}{c|ccccc|ccc}
$\wedge \wedge$ & $T$ & $I$ & $F$ & $\vee$ & $\alpha \wedge$ & $T$ & $I$ & $F$ \\
\hline$T$ & $T$ & $I$ & $F$ & & $T$ & $T$ & $T$ & $T$ \\
$I$ & $I$ & $I$ & $F$ & & $I$ & $T$ & $I$ & $I$ \\
$F$ & $F$ & $F$ & $F$ & & $F$ & $T$ & $I$ & $F$
\end{tabular}

For the partial denotation account, the basic question is how truth-value gaps can be appropriately treated. A solution is offered by the so-called super-valuation technique, which has been first proposed in [van Fraassen 1968]. [Fine 1975] and [Kamp 1975] demonstrate its applicability to the interpretation of vague concepts. [Pinkal 1985] extends the approach to a framework for the description of a variety of indefiniteness phenomena and their interplay with context.

The basic idea of super-valuation is the determination of the value of a complex expression with semantically undefined elements by semantic properties of the set of possible completions, i.e., those classical, complete valuations which are compatible with the given partial one. A complex expression is true under a partial value-assignment $\mathrm{V}$, if and only if it is true in all classical completions of $\mathrm{V}$. It is false, if it is false under all classial completions, and it is undefined otherwise. Take as an example a disjunction of propositional constants, $\mathrm{p} \vee \mathrm{q}$, and a valuation function $\mathrm{V}$ that assigns $\mathrm{T}$ to $\mathrm{p}$, and is undefined for $\mathrm{q}$. $\mathrm{V}$ can be alternatively extended to $\mathrm{V}^{\prime}$ with $\mathrm{V}^{\prime}(\mathrm{q})=\mathrm{T}$, or to $\mathrm{V}^{\prime \prime}$ with $\mathrm{V}^{\prime \prime}(\mathrm{q})=\mathrm{F}$. Now, according to the classical interpretation for disjunction, it holds that $[[q \vee p]]^{\mathrm{V}^{\prime}}=[[q \vee p]]^{\mathrm{V}^{\mathrm{P}}}=\mathrm{T}$; therefore we obtain $[[q \vee p]] \mathrm{V}^{\mathrm{V}}=\mathrm{T}$ by super-valuation. Under the same partial assignment $\mathrm{V}, \mathrm{q} \& \mathrm{p}$ will turn out to be undefined, since $[[q \& p]]^{V^{\prime}}=\mathrm{T}$, but $[[q \& p]]^{\prime \prime}=\mathrm{F}$. If a partial valuation $\mathrm{V}$ is undefined for both $\mathrm{p}$ and $\mathrm{q}$, there are four possible relevant completion alternatives (say, $\mathrm{V}_{1}, \mathrm{~V}_{2}, \mathrm{~V}_{3}, \mathrm{~V}_{4}$ ). Since there is at least a completion making both $p \& q$ and $p \vee q$ true ( say, $V_{1}$ with $\left.V_{1}(p)=V_{1}(q)=T\right)$, and another 
completion rendering both expressions false (say, $V_{4}$ with $V_{4}(p)=V_{4}(q)=F$ ), both $\mathrm{p} \& \mathrm{q}$ and $\mathrm{p} \vee \mathrm{q}$ will turn out to be undefined in V.

Thus, the super-valuation account results precisely in the three-valued truthtables of (13). There is an important difference, however. It becomes apparent if we look at the computation of the value for $A \& B$ and $A \vee B$ in general (i.e., in the case that $\mathrm{A}$ and $\mathrm{B}$ are not independent proposition variables, but arbitrary expressions). Take for example the case where $B=\neg A$, and $[[A]]^{V}$ (and accordingly $[[B]]^{V}$ ) is undefined. Whenever a classical completion $V^{\prime}$ of $V$ yields truth for $A, B$ will be false, according to the classical semantics of negation, and vice versa. Consequently, $A \& B$ will be assigned the value $F$, and $A \vee B$ will be assigned $T$ by the supervaluation, disregarding the fact that both sub-clauses are of indefinite truth value.

The super-valuation account differs in its fundamental properties from the three-valued approach. It is not truth-functional: the value of an expression containing more than one indefinite sub-expressions depends not only on the truth state of its immediate sub-expressions, but also on their form. The deeper reason for the lack of truth-functionality is its sensitive to semantic covariation between different underspecified expressions ([Fine 1975] uses the picture of "penumbral connections" here). Also, there is a difference with respect to the preservation of the classical concepts of validity and entailment: Three-valued approaches make the Law of Excluded Middle as well as the Law of Contradiction contingent formulae because they invariably assign I to conjunctions and disjunctions with indefinite sub-expressions. Since the super-valuation is computed from the values in the classical completion set, it is conservative: there is no chance for a classical tautology or contradiction to become ever false or true, respectively.

It has been extensively discussed, which of the two incompatible approaches provides the more adequate theory of indefiniteness. The important question to be asked is whether the interrelated properties of sensitivity to non-local correlations and conservativity with respect to classical logic are really desirable. ${ }^{4}$ Adherents of many-valued approaches have argued that indefinite truth-value assignment to cases like (14) and (15) be intuitively preferable to the treatment of the sentences as contradiction or tautology, respectively.

John is tall, and he isn't tall

\section{John is tall, or he isn't tall}

An utterance of (14) seems not to be plainly false, given the fact that the vagueness of the adjective tall does not determine a definite truth-value for the sub-clause John is tall, but rather express the information that John is a borderline case of the vague predicate $t$ all. (15) seems to be a reasonable way to express that vague expressions form an exception to the Law of Excluded Middle. Thus the value "indefinite" for (14) and (15) appears to be better justified than the values "false" and "true", respectively. (14) and (15) are problematic evidence for semantic judgments, however. If the sentences are logically determined, their direct interpretation is pragmatically deviant, and it should be expected that hearers reinterpret them in order to make sense out of them, viz. in the sense of (16) and (17), where in some respect and definitely are operators quantifying over contexts/classical completions of the basic partial interpretation.

In some respect, John is tall, in some respect, he isn't

\section{John is de finitely tall, or he is definitely not tall}




\section{John is tall, and Bill isn't tall}

\section{John is tall, or Bill isn't tall (,either)}

The semantically unmarked sentences (18) and (19) form a better test case. Take John and Bill to be of identical height (and take them also to be otherwise similar adult persons, to exclude secondary contextual distortions). Then (18) must be clearly judged as false: Irrespective of where the borderline for tallness is drawn, one of the two conjuncts becomes false. As far as I can see, linguistic evidence speaks definitely in favor of the super-valuation account: not because it preserves tautologies and contradictions of classical logic, in the first place, but because it allows to model covariation between indefinite parts of a meaning structure, which is apparently an essential aspect of natural -language meaning.

Having decided on a super-valuation-based semantics for vague concepts, we can view the status of the formal system in two different ways. One option is to take the system of assignments, consisting of a partial interpretation and alternative completions just as a technical device for the proper interpretation of vague concepts. The other possibility is to take the super-valuation concept as the kernel of a realistic model for contextual meaning variation. This view has been taken in the "precisification semantics" of [Pinkal 1985]. I will shortly sketch its basic ingredients, putting stress on its underlying intuitions and just indicating technical detail.

The basic decision is to consider assignments realistic interpretation options, which are induced by contexts of utterance, rather than technical devices.Then, the standard super-valuation formalism is far too schematic, of course. On the one hand, there must be different partial interpretations corresponding to different kinds and degrees of available context information. On the other hand, classical completions are an ideal which is hardly reachable in natural contexts at all. Accordingly, precisification semantics assumes a set $V$ of partial assignments, which form the "precisification space" of a language or language fragment. The relation " $\leq$ " of "being more precise as" is defined as the subset relation between the members of $V$ : $V \leq V^{\prime}$ if and only if $\operatorname{Dom}(V) \subseteq \operatorname{Dom}\left(V^{\prime}\right)$ and $V(\alpha)=V^{\prime}(\alpha)$ for all $\alpha \in \operatorname{Dom}(V)$. In addition, precisification semantics employs "context spaces", partially ordered sets $\mathrm{C}$ whose elements stand for a certain amount of contextual information, and whose ordering relation $\leq_{\mathrm{c}}$ expresses the ordering of contexts with respect to informativity. A "precisification structure" is defined as a pair $\left\langle V,\left\langle\mathrm{C}, \leq_{\mathrm{c}}\right\rangle\right\rangle$, consisting of a precisification space and a context set, with a homomorphism from $V$ to $\left\langle\mathrm{C}, \leq_{\mathrm{c}}\right\rangle$, corresponding to the fact that precision of an interpretation increases with the growth of context information.

In order to make precisification semantics an equivalent to the standard super-valuation account with respect to meaning composition, the concept of a precisification space must be strengthened, however. In [Pinkal 1985], this is done by the so-called "Principle of Precisification", which consists of two complementary parts. The first part says that whenever for some expression $\alpha$ there are two assignments $V, V^{\prime} \in V$ such that $V(\alpha) \neq V^{\prime}(\alpha)$, then there is a $V^{\prime \prime} \in V$ such that $V^{\prime \prime} \leq \mathrm{V}, \mathrm{V}^{\prime}$, which is undefined for $\alpha$. Intuitively, this constraint corresponds to the statement that every case of contextual variation is a possible source for indefiniteness. This appears to be a plausible assumption, if we take the hearer's view, who may be faced with any kind of deficient context information. The second part of the Precisification Principle states that for every assignment $V \in V$ which is undefined for some expression $\alpha$, there must be assignments $V^{\prime}, V^{\prime \prime} \in V$ such that $\mathrm{V} \leq \mathrm{V}^{\prime}, \mathrm{V}^{\prime \prime}$, which assign $\alpha$ different semantic values. In plain text, the second part of the principle states that every case of indefiniteness is a candidate for alternative contextual specification. This statement seems to be less uncontroversial in the light 
of prototypical vague expressions like colour predicates. But note that it does not imply that there be completely defined assignments $\mathrm{V}$, nor even that a concept occuring in an utterance must have a potential reading which is completely precise reading.

Taken together, both assumptions make up the the Precisification Principle: An expression is of indefinite semantic value, if and only if there are more specific contexts which make it precise in different ways. Technically, the principle imposes a closure condition on the precisification space which renders the precisification approach equivalent to the standard super-valuation account. Intuitively, the principle states an intimate interconnection between context dependence and indefiniteness. They turn out to be the very same phenomenon, looked at from two different points of view: Indefiniteness $i s$ the potential for alternative precisification.

\section{Underspecification and partial interpretation}

The super-valuation framework appears to be a good candidate for a denotational interpretation of underspecified representations, especially in its precisification semantics variant. ${ }^{5}$ The parallels are obvious: Super-valuation employs partial assignments, and provides the possibility to fill in the gaps in alternative ways. Underspecification accounts start with partial specifications of semantic structure, and allow alternative specification in terms of extensions of the constraint set. In the underspecification framework, disambiguation can be done stepwise, by adding constraints one after the other. In precisification semantics, the precisification space may contain chains of partial interpretations ordered by the $\leq$ relation.

For the simple cases of local underspecification (e.g., lexical ambiguities), the connection between underspecified representation and partial interpretation is straightforward. Take sentence (2) with representation (3), which are repeated here as $(20)$ and $(21)$ :

\section{Bill owns a pen}

$$
\left\{\exists x(X(x) \& \text { own }(b, x)), X \in\left\{\text { pen }_{1}, \text { pen }_{2}\right\}\right\}
$$

With respect to denotational interpretation, $\mathrm{X}$ can be treated as a predicate constant which denotes a partial function. The set of possible completions is determined by the constraint $X \in\left\{\right.$ pen $_{1}$, pen 2$\}$. Given that pen and pen 2 have precise, classical denotations corresponding to the sets PEN1 and PEN2, we obtain two complete value assignments $V_{1}$ and $V_{2}$, assigning the values PEN1 and PEN2, respectively, to $X$, and an indefinite interpretation $V \leq V_{1}, V_{2}$, which assigns $X$ the partial function $\varphi$ from individuals to truth values with $\varphi(X)=T$ for members of both PEN 1 and PEN2 (which happens to be the empty set), $\varphi(X)=F$ for individuals in the complement set of PEN $1 \cup$ PEN2, and which is undefined otherwise. Accordingly, sentence (2) is false, if Bill does not stand in the ownership relation to any member of PEN $\cup$ PEN2, it becomes true, if Bill owns both a member of PEN1 and PEN2, and it is undefined otherwise.

The disjunction treatment of underspecified representations discussed at the end of Section 2 (in its wide-scope variant) collapses the truth states "true" and "undefined" to "true", and thus provides a more liberal notion of truth. As mentioned above, this wider notion of truth catches an important point about semantic interpretation in real communication situations, i.e., the hearer's adherence to the principle of charity. The problem is that this kind of cooperative truth assessment is intuitively plausible only for certain types of assertional utterances, 
whereas in the general case a more "objective" notion of truth is in order. The supervaluation approach provides both notions: the concept of "definite truth" - truth in all completions/precisifications of a given partial information - and the more flexible notion of being "acceptable as true" (true in at least one completion/precisification).

\section{Billowns a pen, and John owns a pen, too}

$$
\left\{\exists x(X(x) \& \text { own }(b, x)) \& \exists x(X(x) \& \text { own }(j, x)), X \in\left\{p_{1} n_{1}, \operatorname{pen}_{2}\right\}\right\}
$$

The other problem for the disjunction analysis is that its notions of truth and entailment are far too permissive in general. The flexible truth assessment is independently done for every occurrence of an ambiguous expression, which regularly leads to the selection of mutually incompatible contexts/ disambiguations, as in (22)/(23). The super-valuation account allows to model covariation of parts of the underspecified semantic structure. If entailment is defined in the most straightforward way $-\Gamma \mid=\mathrm{A}$ if $\mathrm{A}$ is true in all classical completions/ precisifications which satisfy $\Gamma$-covariation holds also across the entailment relation, which among other things rules out the unwanted $\mathrm{A} I=\neg \mathrm{A}$ case (see (12) above).

The super-valuation account works well in that it keeps the choice of disambiguations consistent. The problem is that in some cases it seems to do its job too well. Take, e.g., an utterance of (24), where both readings of bank are already present in the context.

\section{The bank is located close to the bank}

It seems not to be a reasonable requirement for sentence (24) that both occurrences of bank covary in their interpretation. Natural language semantics requires a more relaxed notion of covariation for cases like this. Also, in the case of scope ambiguities it is not really clear at all what "different occurences" may be and how covariation should work in general. All in all, it seems that the treatment of vague concepts cannot be generally transferred to ambiguity cases. On the other hand, covariation between occurrences of ambiguous terms definitely occurs, in cases like (22), or yet more mandatory, in the ellipsis construction (25).

\section{Bill owns a pen, and so does John}

We can bring these observations together by assuming that ambiguous expressions are in general represented by different meta-variables. Identical (or co-indexed) meta-variables are introduced only in the case that covariation is explicitly required by the special linguistic construction (e.g., ellipsis, parallel construction, correction). Vague expressions, on the contrary, have a fixed and unambiguous representation with an underspecified denotational interpretation. According to this proposal, the interpretation strictly follows the super-valuation approach for both vagueness and ambiguity phenomena. It has, however, different effects for different kinds of indefiniteness: Different occurrences of a vague expression are bound to covary, since they are represented by the same object-language constant; differences in valuation may occur in certain marked cases, but are due to local context shifts. ${ }^{6}$ Different occurrences of ambiguous expressions covary only in specially designated cases. In general they are independently disambiguated because they have independent representations. The notion of entailment resulting for these cases is more restricted than for the covariation cases: $A$ entails $B$, if every disambiguation of A entails every disambiguation of B. ${ }^{7}$

The picture of an interpreted underspecification formalism is incomplete, so far. Semantic covariation requirements may not only hold for lexical and referential 
ambiguities, but alsō for portions of semantic structure which are underspecified with respect to scope. The classical problem is the parallelism constraint for ellipsis constructions. In (26), the antecedent clause is ambiguous between two scope readings, the reconstructed elliptic clause is, too, but the sentence as a whole has only two rather than four readings, due to the parallelism or structural covariation requirement.

\section{John recommended every student a book, and Bill did, too}

In contrast to the local ambiguity cases, covariation of scoping cannot be represented by just using identical or co-indexed meta-variables, since it involves correspondences of arbitrarily complex pieces of semantic structure. Reyle proposes an extension of his formalism by a mechanism for co-indexing structures ([Reyle 1995], [Reyle 1996]). Co-indexed structures are interpreted by kind of a super-valuation mechanism, where the alternative completions are provided by contexts, which among other things contain complete ordering information for scope-bearing elements. Reyle's proposal is the first one, to my knowledge, which tries to handle the structural covariation problem within an underspecification framework, and he also designs a deduction system, which is sensitive to covariation effects (and definitely an improvement of the one given in [Reyle 1993]). Since he uses quite a bit of technical machinery which is tailored to his UDRT formalism, I am not completely certain about the expressive power and generality of his structural indexing technique.

I would like to indicate a solution for structural covariation which came about as a side-effect of a larger project on semantic analysis of spoken utterances. ${ }^{8}$ Spoken input is often incomplete or incoherent, and therefore a source of another and more radical kind of semantic underspecification. In the most extreme case, the recognized portions of spoken input are just single words. In order to describe the semantic information contributed by such disconnected elements, the standard underspecification framework is extended by second-order meta-variables, which range over semantic operations, rather than expressions of the object representation language. If $X$ is a standard first-order meta-variable, $\alpha$ a piece of semantic structure, and $C$ a second-order meta-variable, the constraint $X=C(\alpha)$ expresses the information that $\alpha$ contributes to the semantics of $X$ in some yet unknown way (i.e., that $X$ is obtained by applying a simple or complex semantic operation to $\alpha$ ). Covariation in ellipsis cases can be naturally expressed with function meta-variables, as shown in (27) for Sentence (26). $X_{A}$ and $X_{E}$ are meta-variables for the semantic representation of the antecedent and the consequent clause, respectively.

$$
\left\{X_{A}=C(\text { john }), X_{E}=C(\text { bill })\right\}
$$

(27) requires that the contrastive elements John and Bill in the construction contribute to the semantics of their respective clauses in the same way. All this is very rough and simplified, of course. Concerning the representation language, e.g., I have not mentioned that the instantiations of the second-order meta-variables must be linear functions (in order to exclude, e.g., constant function which overwrite their arguments). For an adequate treatment of ellipsis, it is necessary to replace the identity requirement for the semantic operations in (27) by a weaker constraint of correspondence, in order to cover the well-known problem cases of ellipsis and binding interaction. I cannot give a more detailed presentation of the second-order underspecification framework in this place. ${ }^{9}$ 


\section{Vagueness, àmbiguity, and the status of representations}

As I have shown in the last section, vagueness and ambiguity can basically be treated alike, on the level of denotations. In order to account for their different behaviour with respect to covariation, I have proposed a solution, which makes essential reference to a level of representation: Vague expressions are represented by unique constants with partial denotations, ambiguous expressions by meta-variables ranging over different constants of the representation language. This assumption seems also to correspond well to some other differences between the phenomena: Ambiguous expressions have a finite, at least a discrete set of readings corresponding to possible instantiations of meta-variables with objects of the representation language, where vague expressions tend to allow a continuous range of precisifications. Ambiguous expressions always allow complete disambiguation, whereas it is a significant feature of vague expressions that they keep an irreduible margin of imprecision. Disambiguation of ambiguous expressions requires the choice among alternative readings, which mutually exclude each other. Precisification of vague expressions takes place by reduction of the borderline area of indefiniteness in more or less arbitrary ways. Thus, for two different precisifications $V$ and $V$ ' of a vague predicate there is always a (less indefinite and) more general one, which includes the positive application domain of $\mathrm{V}$ and $\mathrm{V}^{\prime}$ in its positive domain. This difference is the basis for the standard ambiguity tests (see, e.g., [Lakoff 1973]); I will refer to the distinction somewhat sloppily as the "most-general-reading criterion" in the following.

There is one more, intuitively prominent, difference between typical kinds of vagueness and ambiguity. It has been often observed and described with different metaphors, for example in the following two citations:

"Vague terms are only dubiously applicable to marginal objects, but an ambiguous term such as "light" may be at once clearly true of various objects (such as dark feathers) and clearly false of them." [Quine 1960]:128

"Vague and ambiguous sentences are subject to similar truth-conditions; a vague sentence is true if true for all complete precisifications; an ambiguous sentence is true if true for all disambiguations... However, how we grasp the precisifications and disambiguations, respectively, is very different. ...disambiguations are distinguished; to assert an ambiguous sentence is to assert, severally, each of its disambiguations. .... precisifications are extended from a common basis and according to common constraints; to assert a vague sentence is to assert, generally, each of its precisifications. Ambiguity is like the super-imposition of several pictures, vagueness like an unfinished picture, with marginal notes for completion." [Fine 1975]:282f.

In vague expressions, the underspecified basic interpretation is cognitively prominent, in ambiguous expressions it is the disambiguations. Accordingly, underspecified interpretations of ambiguous sentences occur, but they occur as interpretations in the hearer's underspecified context only, and are of a rather instable and volatile character, as opposed to the stable and unmarked vaguenesstype indefiniteness. Again, this fits well to the assumed different representational status of vagueness and ambiguity - given that the level of representation has an independent cognitive reality. One might even conjecture that the classical distinction between vagueness and ambiguity based on the notion of the unity of a word (see, e.g., [Lyons 1977]) coincides with the existence of a single representational unit, i.e., an object of the representation language. 
The problem is that not all of the distinctions between vagueness and ambiguity coincide. Just for completeness, I mention that the two criteria for vagueness diverge: The continuity-discreteness distinction draws a narrower boundary than the most-general-reading criterion. It is the continuity cases of vagueness which are the really hard ones for a logic-based account of meaning, since continuity of the precisification space causes practical undelimitability and gives rise to the so-called heap paradox. ${ }^{10}$

More importantly, the two criteria which are the most relevant ones for the notion of ambiguity - (non-)availability of a most general reading and (non)availability of a stable underspecified basic reading - are not co-extensional. Take the adjective fast, which is an example for the systematic ambiguity between a stage level and an individual-level, dispositional reading.

The Porsche is fast, and the $2 \mathrm{CV}$ is, too

\section{There are two fast cars in the street}

If you are in a situation where a Porsche is parked in a residential street, and a $2 \mathrm{CV}$ passes by with a speed of $50 \mathrm{mph}$, it would be strange to utter (28) or (29). There is no common most general reading covering the stage and individual level uses of fast. Therefore, fast is clearly ambiguous rather than vague. However, in cases where the ambiguity is irrelevant for the truth assessment of the utterance, the underspecified use of the predicate fast is perfectly fine. Sentence (30), when uttered with emphasis about a Porsche which rushes along the German Autobahn with $130 \mathrm{mph}$, assigns both readings of fast at the same time, and there is no need for disambiguation.

\section{This is a fast car!}

Another interesting case where unmarked use of the underspecified semantics is possible are scope ambiguities. In fact, I gave an example already in (1) of Section 1. Speakers often seem to use scope-ambiguous utterances without having a certain reading in mind. But quantifiers also pass the ambiguity test: It is a well-known fact that there is a covariation requirement for scope in ellipsis constructions. Moreover, there is not even in principle a possibility to represent scope-underspecified structures by a unique, only denotationally indefinite object-language representation, because of the non-local character of scope ambiguities.

Obviously, the ambiguity criteria of a widest reading and a stable basic reading do not coincide, and the distinction cannot easily be related either to the denotational structure or to the representational status of ambiguous concepts. As always in cases where no straightforward semantic means are available to make a distinction, it is tempting to try a general pragmatic explanation. In fact, the examples considered suggest a pragmatic answer to the problem in terms of Gricean principles: "Strong" cases, which do not allow stable underspecified representations, like pen, bank, and referential ambiguities, have disjoint extensions. Therefore, sentences get a chance to become unequivocally true only in the case that they are disambiguated. On the other hand, "weak" cases of ambiguity like fast and quantifier constructions may well be true under both readings, and accordingly do not need immediate precisification.

However, this cannot be the answer, either. As pointed out above, Sentence (20) can be definitely true, but this does not give its basic underspecified interpretation the same status as (1) or (30). I will not discuss the possibility to state a pragmatically relevant difference between the pen and the fast cases here, but move on to another example, which provides yet stronger evidence against a pragmatic 
explanation. It concerns scope underspecification again, and was brought to my attention by Uwe Reyle.

\section{Every student didn't pay attention}

\section{Every student overlooked a mistake}

There is no structural difference between sentences $(31)(=(5))$ and $(32)$ on the level of denotations: Both are ambiguous between two readings, and in both cases one reading is included in the other, which should make either sentence a perfect candidate for weak ambiguity. Also, there is no difference on the representation level, if available techniques for underspecified representation are used. Nevertheless, the underspecified reading of (32) seems to be a cognitively easily graspable weak ambiguity case, whereas (31) has a homonymic, strongly ambiguous character.

To sum up, there seems to be no convincing way to explain the differences in the behaviour of ambiguous expressions in pragmatic terms. We have to accept the fact that the strong-weak distinction is not a matter of use. The range of expressions or construction types which have different and mutually exclusive specific readings subdivides into cases where there is a cognitively available common underspecified reading, as well as cases where it isn't. But there is no general property of the denotational interpretation of these expressions which may be used to distinguish between strong and weak ambiguity cases. This draws the attention directly to the level of representation. The difference between polysemy and homonomy for lexical ambiguities could in principle be explained in terms of the availability of a common underspecified object-language representation unit. This would leave us with the task however to find a separate explanation for the ambiguous character of fast (concerning the most-general-reading criterion).

The case of scope ambiguity is harder: There seem to be strong and weak cases. None of them can have a unique object-language representation, all of them have similar description-language representations. On the one hand, this implies that underspecified representations are not just abbreviatory notations, but are the only way to express certain "cognitively real" entities of natural-language meaning structure. On the other hand, the fact that a piece of semantic information is expressible in the underspecification language is as irrelevant to the strong-weak distinction as the question of expressibility in the object language itself is.

There must be something relevant in word meaning beyond denotations and logical representations. In a way, it seems to be some common conceptual substance behind the different readings, which constitutes the unity of an expression. This could be a prototype for colour adjectives and concrete common nouns, the "concept of fastness" which goes into the semantics of the stage and the individual-level reading of fast, or, to take an example from the area of referential underspecification, the "speaker's involvement" for the plural pronoun we, which has perfectly accessible underspecified readings, even the extreme one where only the speaker is definitely included.

Another interesting perspective is suggested by the pair of scope-ambiguous expressions $(31) /(32)$. The weak-strong difference corresponds to a difference in deductive properties. As Reyle argues in [Reyle 1993] and [Reyle 1995], the extension of standard deduction techniques to underspecified representations makes a case distinction necessary between the status of sub-expressions. The rules of direct deduction are sensitive to the question of whether an expression occurs in an upward or a downward monotonic position. Since the scope ordering is relevant for the monotonicity status, and scope can be underspecified, the monotonicity feature may be underspecified, as well. In this case, direct deduction is impossible, and the 
only thing left to do is to spell out the ambiguity, and do deduction separately on the readings. The system described in [Reyle 1995] models this interplay between underspecified deduction and partial resolution steps. Now, the weak ambiguity case (32) is unambiguously upward monotonic and therefore allows direct deduction, whereas in the strong ambiguity case (31) is a case where the universal quantifier is of uncertain monotonicity status because it can be ordered either below or above the negation operator; therefore (31) cannot be directly processed, but must be disambiguated first. The cognitive reality of a semantic object may be dependent on the fact whether the basic reading allows direct processing.

All this is highly speculative and vague, of course. What we can state with certainty about the interrelation between logical representation and cognitive reality, is that we are still in a stable state of informational underspecification.

\section{Endnotes}

${ }^{1}$ [Pinkal 1995] is a revised and extended English version of [Pinkal 1985]: The chapters $1-7$ are identical in content; the last chapter is completely revised. I will use [Pinkal 1985] as reference, when speaking about old results,

2 Among them are Quasi-Logical Form developed at SRI Cambridge as part of the Core Language Engine (QLF, [Alshawi/Crouch 1992]), Uwe Reyle“s UDRT ([Reyle 1993]); Labelled Underspecified Discourse Representation (LUD, [Bos 1995]) and Minimal Recursion Semantics (MRS, [Copestake/Sag 1995], [Egg/Lebeth 1995]), which serve as semantic representation formalisms in the MT project Verbmobil, Massimo Poesio's DRT-based system [Poesio 1994], and a formalism by Reinhard Muskens, based on his Compositional DRT [Muskens 1995], and Jan van Eijck's logic of ambiguation [Cooper et al. 1996].

3 The differences between the 3-valued logics of [Lukasiewicz 1930], [Kleene 1952], [Blau 1978] concern the treatment of implication and quantifiers; they are discussed in detail in [Pinkal 1985]. These differences are not relevant for the argument of this paper. Also, I will not consider the option of indefinitely-valued logics (like fuzzy logic), which play an important role in non-linguistic computational applications.

${ }^{4}$ For a detailed presentation and discussion of the arguments see [Pinkal 1985], Chapter 4. - I leave out the computational complexity argument here, which has played an important role in computational semantics. To be sure, the supervaluation account is more complex, but "non-truthfunctional" does not mean "noncompositional". If we decide to assign a sentence not a truth-value, but the set of its verifying classical completions as denotation, then the interpretation of conjunction and disjunction is given in a straightforward compositional manner in terms of union and intersection, respectively. In general, the denotation of every expression can be defined as a function from the set of possible completions (or contexts, see below) to appropriate extensions.

5 Using super-valuation for the interpretation of underspecified representations was first proposed in the QLF context by [Alshawi/Crouch 1992]; see also [Cooper et al. 1994] and [Alshawi 1996]. The approaches discussed in [van Deemter 1996] and [Reyle 1995], [Reyle 1996] also come close to the super-valuation idea.

${ }^{6}$ The case of predicative scalar adjectives like tall is maybe best described by a combination of underspecification (i.e., underspecification of the modified predicate, represented by the meta-variable $X$ in tall $(X)$, and an amount of vagueness which remains in every instantiation of the predicate.

${ }^{7}$ This is the "cautious" entailment concept proposed by Jan van Eijck in [Cooper et al. 1996], and discussed as one possible variant ( $\forall \forall$ without coherence) in [van Deemter 1996]. Kees van Deemter also tries to catch a wider concept of coherence 
with logical means, i.e., the fact that the choice of identical readings for two occurrences of an ambiguous term is in general preferred, if it allows a sensible interpretation. I tend to take this as a pragmatic coherence phenomenon, and to separate it from the cases of linguistically required covariation.

${ }^{8}$ CHORUS - "Constraint-based Higher-order Representation of Underspecified Semantic Information", in the Special Research Division "Ressource-Adaptive Cognitive Processes" of the DFG (German Science Foundation)

${ }^{9}$ I would like to mention, though, that the framework takes care of all phenomena of ellipsis and scope interaction known from the literature, including antecedentcontained deletion and the Hirschbuehler cases.- For a description of the representation language (USDL : Underspecified Semantic Description Language), see [Pinkal 1995a].

${ }^{10} \mathrm{~A}$ detailed discussion is found in Chapter 8 of [Pinkal 1995].

\section{References}

Alshawi, H., and R. Crouch (1992). Monotonic semantic interpretation. In Proceedings of the 30th Annual Meeting of the Association for Computational Linguistics, 32-38.

Alshawi, H. (1996). Underspecified First Order Logics. In Semantic ambiguity and underspecification, ed. K. van Deemter and S. Peters, 145-158. Stanford, California: CSLI Publications.

Black, M. (1937). Vagueness: An exercise in logical analysis, Philosophy of Science 4: $427-455$.

Blau, U. (1978): Die dreiwertige Logik der Sprache. Berlin: de Gruyter.

Bos, J. (1995): Predicate logic unplugged. In Proceedings of the 10th Amsterdam Colloquium.

Cooper, R. et al. (1994). Describing the approaches. FraCaS deliverable D8, December 1994. FraCaS, A Framework for Computational Semantics, LRE $62-051$.

Cooper, R. et al. (1996). Building the framework. FraCaS deliverable D15, January 1996. FraCaS, A Framework for Computational Semantics, LRE $62-051$.

Copestake, A., D. Flickinger, R. Malouf, S. Riehemann, and I. Sag (1995). Translation using minimal recursion semantics. In Proceedings of the Sixth International Conference on Theoretical and Methodological Issues in Machine Translation. University of Leuven. Belgium.

van Deemter, K. (1996). Towards a logic of ambiguous expressions. In Semantic ambiguity and underspecification, ed. K. van Deemter and S. Peters, 203235. Stanford, California: CSLI Publications.

Egg, M., and K. Lebeth (1995). Semantic underspecification and modifier. In Integrative Ansätze in der Computerlinguistik (DGfS/CL '95), ed. J. and R. Wiese, 19-24. Seminar für Allgemeine Sprachwissenschaft: Düsseldorf.

Fine, K. (1975). Vagueness, truth, and logic. Synthese 30: 265-300.

van Fraassen, B. (1968). Presupposition, implication, and self-reference. The Journal of Philosophy: 136-152.

Kamp, H. (1975). Two theories about adjectives. In Formal Semantics of Natural Language, ed. E. Keenan. Cambridge: Cambridge University Press.

Kamp, H. (1981). A theory of truth and semantic representation. In Formal Methods in the Study of Language, ed. J. Groenendijk et al. Mathematisch Centrum, Amsterdam.

Kleene, S. (1952). Introduction to Metamathematics, Amsterdam, North Holland. 
Kuschert, S., M. Kohlhase, and M. Pinkal (1995). A type-theoretic semantics for Lambda-DRT. In Proceedings of the Tenth Amsterdam Colloquium.

Lakoff, G. (1973). Hedges: A study in meaning criteria and the logic of fuzzy concepts, Joumal of Philosophical Logic 2: 458-508.

Lukasiewicz, J. (1930). Philosophische Bemerkungen zu mehrwertigen Systemen des Aussagenkalküls In Comptes rendus des séances de la Société des Sciences et des Lettres de Varsovie. Classe III 23: 51-77.

Lyons, J. (1977). Semantics. 2 vols., Cambridge University Press.

Montague, R. (1973). The proper treatment of quantification in ordinary English. In Approaches to Natural Language, ed. J. Hintikka. Dordrecht: Reidel.

Muskens, R. (1995). Order-independence and underspecification. In Proceedings of the Tenth Amsterdam Colloquium.

Pinkal, M. (1985). Logik und Lexikon-Die Semantik des Unbestimmten. Berlin und New York: Walter de Gruyter.

Pinkal, M. (1995). Logic and lexicon. A revised and extended version of Pinkal 1985. Dordrecht: Kluwer Academic Publishers.

Pinkal, M. (1995a). Radical underspecification. In Proceedings of the Tenth Amsterdam Colloquium.

Poesio, M. (1994). Discourse interpretation and the scope of operators. Doctoral dissertation. University of Rochester, Department of Computer Science. Rochester, NY.

Reyle, U. (1993). Dealing with ambiguities by underspecification: construction, representation and deduction. Journal of Semantics 10: 123-179.

Reyle, U. (1995). On reasoning with ambiguities. In Seventh Conference of the European Chapter of the Association for Computational Linguistics Proceedings of the Conference. ACL, Dublin, 1-8.

Reyle, U. (1996). Co-Indexing Labeled DRSs to Represent and Reason with Ambiguities. In Semantic ambiguity and underspecification, ed. K. van Deemter and S. Peters, 55-76. Stanford, California: CSLI Publications. 